\title{
Damage accumulation in thin ruthenium films induced by repetitive exposure to femtosecond XUV pulses below the single- shot ablation threshold
}

\author{
IgOR A. MAKHOTKIN ${ }^{1 *}$, IGOR MILOV ${ }^{1}$, JAROMIR ChalupskÝ ${ }^{2}$, KAI TIEDTKE ${ }^{3}$, \\ HaRTMUt ENKISCH ${ }^{4}$, Gosse de VRIES ${ }^{5}$, Frank SCHOLZE ${ }^{6}$, FranK \\ SieWert ${ }^{7}$, Jacobus M. Sturm ${ }^{1}$, Konstantin V. NikolaeV ${ }^{1}$, Robbert W. \\ E. van de KruiJs ${ }^{1}$, MARK A. Smithers ${ }^{8}$, Henk A.G.M. van Wolferen ${ }^{8}$, \\ ENRICO G. KEIM ${ }^{8}$, ERIC LOUIS ${ }^{1}$, IWANNA JACYNA ${ }^{9}$, MAREK JUREK ${ }^{9}$, \\ Dorota KLINGER ${ }^{9}$, JeRzY B. PELKA ${ }^{9}$, LIBOR JUHA ${ }^{2,10}$, VĚrA HÁJKOVÁ ${ }^{2}$, \\ VOJTĚCH VOZDA ${ }^{2,11}$, TOMÁŠ BURIAN ${ }^{2,10}$, KAREL SAKSL $^{2,12}$, BART FAATZ ${ }^{3}$, \\ Barbara Keitel ${ }^{3}$, Elke PlönJes ${ }^{3}$, Siegfried Schreiber ${ }^{3}$, Sven \\ Toleikis ${ }^{3}$, Rolf Loch ${ }^{3}$, Martin HermanN ${ }^{4}$, Sebastian Strobel ${ }^{4}$, \\ RILPHO DONKER ${ }^{5}$, TOBIAS MEY ${ }^{13}$ AND RYSZARD SOBIERAJSKI ${ }^{9}$.
}

\footnotetext{
${ }^{1}$ Industrial Focus Group XUV Optics, MESA+ Institute for Nanotechnology, University of Twente, Drienerlolaan 5, Enschede, 7522 NB, The Netherlands

${ }^{2}$ Institute of Physics, Academy of Sciences of the Czech Republic, Na Slovance 2, Prague 8, 182 21, Czech Republic

${ }^{3}$ Deutsches Elektronen-Synchrotron DESY, Notkestrasse 85, Hamburg, 22607, Germany

${ }^{4}$ Carl Zeiss SMT GmbH, Rudolf-Eber-Strasse 2, Oberkochen, 73447, Germany

${ }^{5}$ ASML Netherlands B.V., P.O. Box 324, Veldhoven, 5500 AH, The Netherlands

${ }^{6}$ Physikalisch-Technische Bundesanstalt, Abbestr. 2-12, Berlin, 10587, Germany

${ }^{7}$ Helmholtz Zentrum Berlin für Materialien und Energie, Albert-Einstein-Str. 15, Berlin, 12489, Germany

${ }^{8}$ MESA + NanoLab, University of Twente, De Achterhorst 75, Enschede, 7522 EA, The Netherlands

${ }^{9}$ Institute of Physics, Polish Academy of Sciences, Al. Lotników 32/46, Warsaw, PL-02-668, Poland

${ }^{10}$ Institute of Plasma Physics, Academy of Sciences of the Czech Republic, Za Slovankou 3, Prague 8, 182 00, Czech Republic

${ }^{11}$ Institute of Physics of Charles University, MFF, Ke Karlovu 5, Prague 2, Czech Republic

${ }^{12}$ Institute of Materials Research, Slovak Academy of Sciences, Watsonova 47, Košice, 040 01, Slovak Republic

${ }^{13}$ Laser-Laboratorium Göttingen e.V., Hans-Adolf-Krebs-Weg 1, Göttingen, 37077, Germany

*i.makhotkin@utwente.nl
}

\begin{abstract}
The process of damage accumulation in thin ruthenium films exposed to multiple femtosecond XUV free electron laser (FEL) pulses below the critical angle of reflectance at the Free electron LASer facility in Hamburg (FLASH) was experimentally analyzed. The multishot damage threshold is found to be lower than single-shot damage threshold. Detailed analysis of the damage morphology and its dependence on irradiation conditions justifies the assumption that cavitation induced by the FEL pulse is the prime mechanism responsible for multi-shot damage in optical coatings.
\end{abstract}

(C) 2018 Optical Society of America under the terms of the OSA Open Access Publishing Agreement

\section{Introduction}

The development of high peak brilliance, high repetition rate free electron laser (FEL) light sources operating in the XUV and X-ray spectral regime such as FLASH, FLASH 2, European XFEL and LCLS leads to increased practical interest in the durability of thin films exposed to 
a large number of pulses. FEL optical elements such as mirrors or beam-stoppers should be designed considering the limitation on durability to the FEL radiation together with requirements on high optical performance of the materials. Avoiding of FEL-induced damage is also critical when long exposures of thin film samples are foreseen during FEL experiments. Examples of such experiments can be found in time resolved studies of surface chemical reactions with pump-probe X-ray diffraction techniques [1].

Only recently the first papers on resistivity of metal films to multi-shot X-ray radiation were published [2, 3]. Earlier, Hau-Riege et.al. [4] investigated $\mathrm{B}_{4} \mathrm{C}$ coatings. In both cases it was observed that the multi-shot damage threshold is slightly lower than the single-shot one, but no detailed analysis of the nature of multi-shot damage was yet reported. Within the extreme ultraviolet (XUV) wavelength range multi-shot damage studies of bulk $\mathrm{Si}$ samples were reported by Sobierajski et al. [5]. The prime damage mechanism was identified as crossing the melting threshold due to heat accumulation in the vicinity of the Si surface.

In this work we investigate the multi-shot damage threshold (MSDT) for Ru coatings exposed to different number of $100 \mathrm{fs}$ pulses at $13.5 \mathrm{~nm}$ wavelength generated with different repetition rates. We present the dependence of the MSDT on the repetition rate and the number of pulses. The influence of irradiation conditions, namely the number of pulses and grazing incidence angle, on the morphology of the damaged spots is investigated. Mechanisms responsible for the observed damage phenomena are suggested, although detailed atomistic simulations are required to confirm the proposed mechanisms.

Understanding the multi-shot damage of Ru films has a practical relevance. Since Ru has a high reflection coefficient for the extreme ultraviolet and soft X-ray wavelength ranges, and has a low oxidation rate, it is a very attractive material for reflective coatings. This work is a continuation of a series of studies of optics durability exposed to FEL radiation below the single-shot damage threshold published here [6] and a detailed analysis of the nature of single shot damage discussed here [7].

\section{Experimental}

For this study, polycrystalline Ru coatings of $50 \mathrm{~nm}$ thickness were deposited on to superpolished silicon substrates using magnetron sputtering in Ar atmosphere. The thickness of the $\mathrm{Ru}$ layer was determined by X-ray reflectivity measurements. Exposure of the Ru films was performed at the BL2 beamline of the FLASH facility [8]. Details of the experiment can be found elsewhere [6,9]. For irradiations we used 100 fs XUV pulses with a wavelength of 13.5 $\mathrm{nm}$. The light was p-polarized with respect to the sample surface. We used the multi-shot irradiation mode where FLASH generated one pulse train per 0.1 second [10]. The maximum duration of a pulse train was $400 \mu$ s and the maximum repetition rate within the pulse train was $1 \mathrm{MHz}$. During the experiment we controlled the number of pulses within the pulse train by reducing the duration of the pulse train. It was also possible to reduce the repetition rate while keeping the pulse train duration constant, and thus reducing the number of pulses in the pulse train.

The determination of the MSDT was performed using Liu's method [11-13] and fluence scan method $[9,14]$ based on measuring damaged areas with differential image contrast (also referred to as Nomarski) microscopy. The dependence of the damage threshold on the repetition rate and the number of pulses was studied using irradiations at $3.17^{\circ}$ grazing incidence with an effective area of the beam $[14,15]$ on the sample surface of $40546 \mu \mathrm{m}^{2}$. Development of the damage morphology was studied by analysis of damage craters caused by different number of pulses arriving at $20^{\circ}$ grazing incidence at a fixed average fluence per pulse using a focused beam with an effective area on the sample surface of $120 \mu \mathrm{m} 2$. The XUV reflectivity values at $3.17^{\circ}$ and $20^{\circ}$ degrees were measured to be $95.5 \%$ and $68 \%$, respectively [16]. In both cases only the evanescence wave penetrates into the film resulting in a comparable penetration depth for these different angles of incidence $-2.5 \mathrm{~nm}$ and $3.5 \mathrm{~nm}$, respectively. For Ru, the critical reflection angle for XUV radiation at a wavelength of $13.5 \mathrm{~nm}$ is $27^{\circ}$. 
For a consistent comparison of the damage threshold values at different irradiation conditions, all analyses is performed in terms of absorbed fluence. It is calculated according to the formula Fabs $=(1-\mathrm{R}) * \mathrm{E} / \mathrm{A}$ where $\mathrm{E}$ is the total energy of the pulse, $\mathrm{A}$ is the effective area of the beam on the sample surface determined according to [15], and $\mathrm{R}$ is the reflectivity coefficient at the corresponding exposure conditions. The total energy of the pulse $\mathrm{E}$ was measured with gas monitor detector described by Tiedtke et.al. [17].

\section{Results and discussions}

First, we present the results of multi-shot damage experiments of $50 \mathrm{~nm} \mathrm{Ru}$ films performed at a grazing incidence of $3.17^{\circ}$. The $\mathrm{Ru}$ films were exposed to single pulse trains, consisting of a different number of pulses from 50 to 400 at a fixed repetition rate of $1 \mathrm{MHz}$. Another set of exposures was performed for a fixed number of pulses in a pulse train (100 pulses), but upon varying the repetition rate (time interval between pulses in a train). The measured damage threshold values are summarized in Table 1.

Table 1. Dependence of the multi-shot damage threshold in $\mathrm{mJ} / \mathrm{cm} 2$ (absorbed fluence) on repetition rate and number of pulses in a pulse train at $3.17^{\circ}$ grazing incidence. The error in the threshold determination is in the order of $20 \%$.
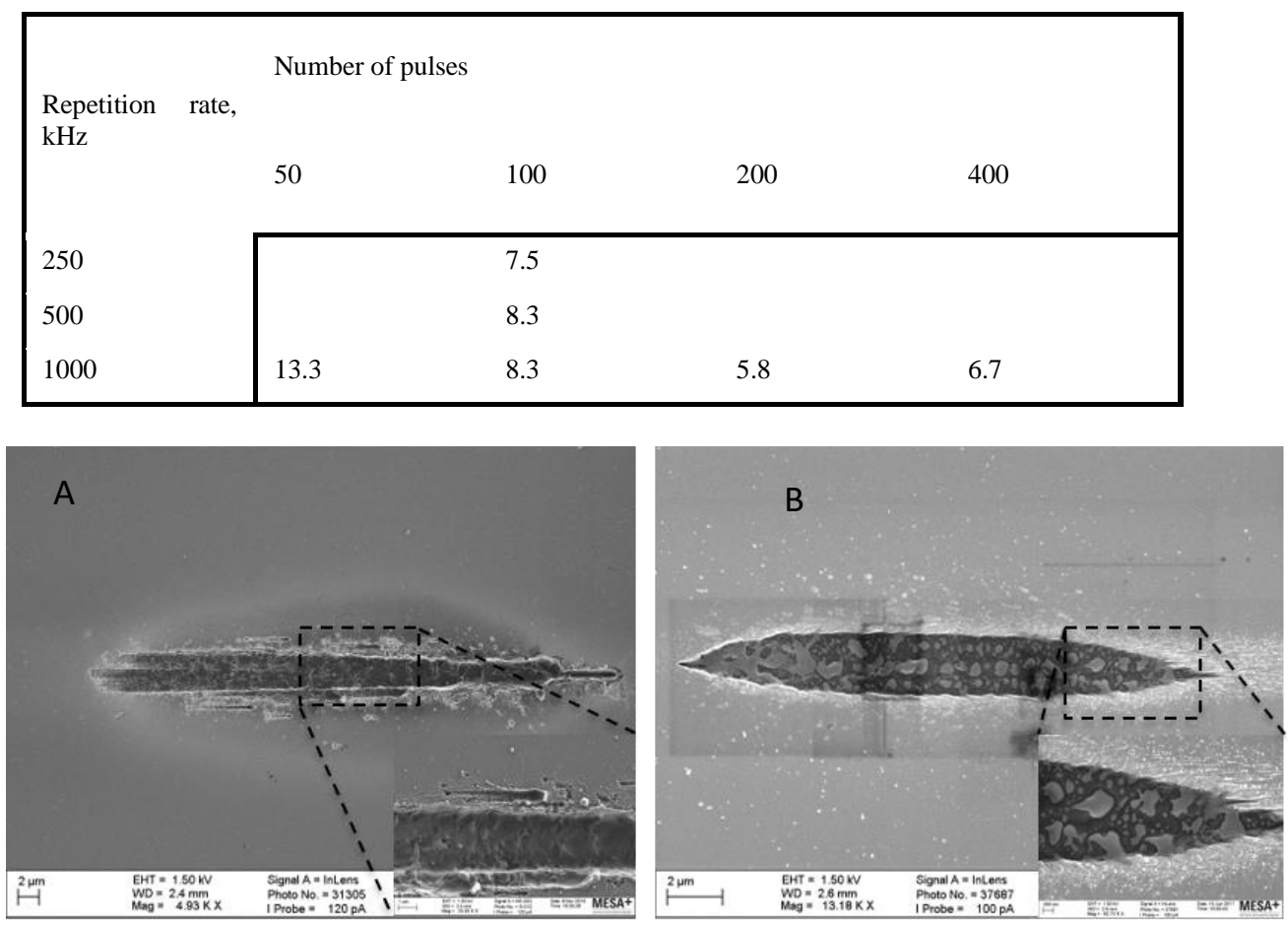

Fig. 1 HR-SEM image of a damaged spot produced with a) pulse train consisting of 50 femtosecond XUV pulses with $1 \mathrm{MHz}$ repetition rate at $3.17^{\circ}$ grazing incidence angle and b) 400 femtosecond XUV pulse trains at $20^{\circ}$ grazing incidence. Each pulse train consisted of 400 pulses. Pulse train repetition rate was $10 \mathrm{~Hz}$, while pulse repetition rate within a pulse train was $1 \mathrm{MHz}$.

As one can see, the MSDT does not depend on repetition rate within the error margin which in our case was around $20 \%$. This suggests that heat accumulation is unlikely to be the damage mechanism for the given experimental conditions. This is in contrast to the previously reported work of Sobierajski et al. [5], where heat accumulation in bulk Si exposed to similar radiation condition but at a normal incidence was found to be as the main mechanism responsible for multi-shot damage caused by XUV femtosecond FLASH laser pulses. Ru, being a metal, has a 
high thermal conductivity, which guarantees that the energy that is absorbed at the surface, diffuses through the entire Ru layer into the Si substrate mostly before the next pulse in the train arrives.

Another observation is the decrease of the damage threshold upon increasing the number of pulses to a fixed repetition rate of $1 \mathrm{MHz}$ stabilizing at 200 pulses and more. This result suggest together with the independence of the MSDT on the repetition rate described above, that there is an accumulation of an irreversible process. Increase of MSDT with the decrease of the number of pulses from 100 to 50 indicates that damage caused by a smaller number of pulses with low fluence is not be observed, and that a large fluence of individual pulse is needed to cause detectable damage.

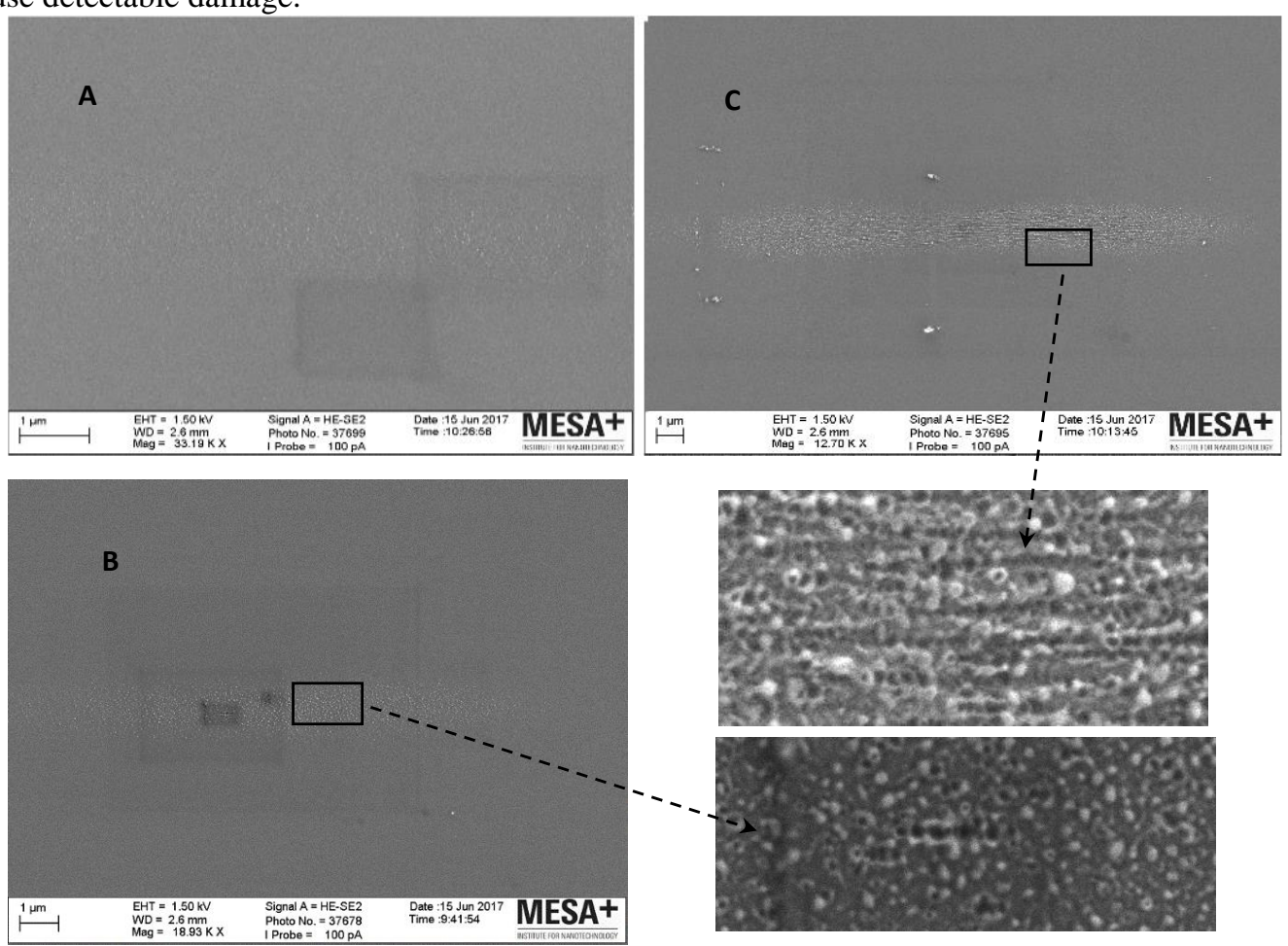

Fig. 2 HR-SEM images of damage morphologies caused by 1600 (A) 209 (B) and 9200(C) femtosecond XUV pulses at $20^{\circ}$ grazing incidence condition.

In order to understand the processes responsible for the multi-shot damage of $\mathrm{Ru}$, we performed ex-situ analysis of the damage morphology by means of high-resolution scanning electron microscopy (HR-SEM) and transmission electron microscopy (TEM). Additionally, we compared the damage morphologies obtained at $3.17^{\circ}$ and $20^{\circ}$ grazing incidence to study the influence of different angles of incidence. The HR-SEM image of a damaged spot exposed to a single pulse train that consisted of 50 pulses with $1 \mathrm{MHz}$ repetition rate at $3.17^{\circ}$ grazing incidence is shown in Fig. 1(a). The mean value of the fluence per pulse was $15 \mathrm{~mJ} / \mathrm{cm}^{2}$, which is close to the measured damage threshold value in table 1 , which is $13.3 \mathrm{~mJ} / \mathrm{cm}^{2}$. The damaged spot has a total area of $\sim 320 \mu \mathrm{m}^{2}$ and can be characterized as a deep crater penetrating through the Ru layer into the Si substrate. The fact that the crater depth exceeds the thickness of the Ru layer is verified by imaging, using an energy selective backscattered detector (not shown), which is sensitive to elemental composition of a surface, confirming that $\mathrm{Si}$ is at the bottom of the crater. No other significant surface modifications around the craters, such as increased roughness or cracks, were detected by means of HR-SEM. Fig.1(b) shows a crater resulting from irradiation of the Ru film with a much larger number of pulses, namely 160 thousand (400 
pulse trains of 400 pulses each at a repetition frequency of $1 \mathrm{MHz}$ ), but performed at $20^{\circ}$ grazing incidence and a mean value of fluence per pulse of $12.5 \mathrm{~mJ} / \mathrm{cm}^{2}$. From the color contras we can conclude that the crater shown in Fig. 1(b) has smooth silicon surface with Ru droplets on top. Fig. 1 shows that, although both exposures are carried out with a fluence close to the damage threshold, a much smaller number of pulses arriving at $3.17^{\circ}$ causes comparable damage to the one caused by pulses arriving at $20^{\circ}$ grazing incidence.

To elaborate on the mechanism of accumulation of damage we study the development of damage with increasing number of pulses for irradiation at $20^{\circ}$ grazing incidence angle. The following exposures were analyzed: 209 individual pulses generated at $5 \mathrm{~Hz}$ repetition rate, and 4 and 23 pulse trains, generated with $10 \mathrm{~Hz}$ repetition rate. Each train in this experiment consisted of 400 pulses generated with $1 \mathrm{MHz}$ repetition rate. The damage threshold value, determined by Liu's method, for one such pulse train at $20^{\circ}$ was $14 \pm 3 \mathrm{~mJ} / \mathrm{cm}^{2}$.

The mean value of the fluence per pulse in the irradiation was $12.5 \mathrm{~mJ} / \mathrm{cm}^{2}$ which is slightly below the MSDT. Only surface modifications of the Ru layer were detected (see Fig. 2). TEM and AFM measurements of the damaged spots (not shown) confirmed that only the Ru layer is modified. All three damaged spots presented in Fig. 2 can be characterized as increased surface roughness, although the degree of roughening is different. Surprisingly, the surface roughness is considerably lower after irradiation with 1600 pulses, than after 209 individual pulses, whereas the opposite is expected.

The possible explanation of this contradiction is the random character of the pulse fluences within the irradiation. We should note that because of the self -amplified spontaneous emission (SASE) operation of the FEL, the fluences of individual pulses varied from 1 to $30 \mathrm{~mJ} / \mathrm{cm} 2$. The histograms of absorbed fluences per pulse for irradiations with 209, 9200 and 1600 pulses are shown in Fig. 3.

The solid lines in Fig. 3 show the number of pulses within the exposure sequence that have a fluence higher than the $\mathrm{x}$ coordinate value. Based on such analysis (see the magnified image in Fig. 3) one can see that there are more high-fluence pulses (more than $20 \mathrm{~mJ} / \mathrm{cm}^{2}$ of absorbed fluence) in the case of irradiation with 209 pulses than in the case of 1600 pulses. If we assume that there is a damage threshold in the range of $20-25 \mathrm{~mJ} / \mathrm{cm}^{2}$ it will lead to the conclusion that with 209 pulses, the Ru film was exposed to a larger number of pulses that can cause damage than in the 1600 pulses case, explaining the larger damage with 209 pulses.

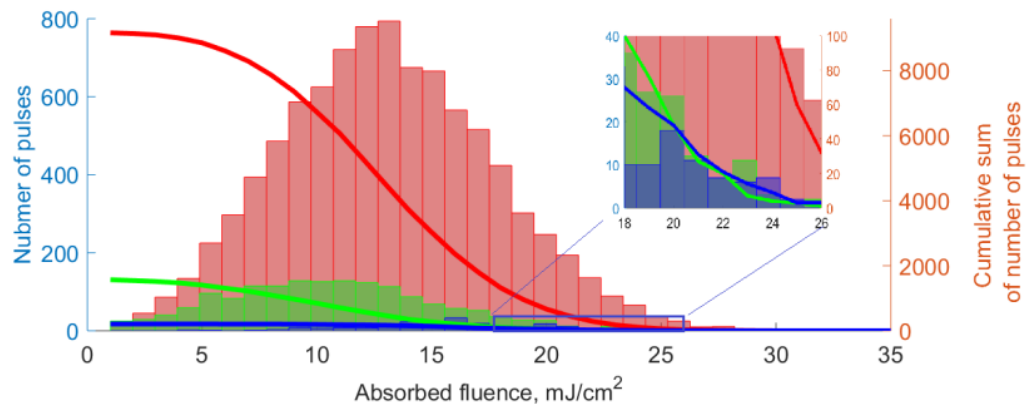

Fig. 3 Histograms of fluences for irradiation containing 209 (blue), 1600 (green) and 9200 (red) pulses at 20 degrees grazing incidence. The solid lines correspond to the cumulative sum of the number of pulses with a fluence equal to and higher than the $\mathrm{x}$ coordinate value calculated by the formula: $\sum_{F=F(x)}^{F M a x} N\left(F_{a b s}\right)$, where $\mathrm{F}$ is the absorbed fluence, and $\mathrm{N}\left(\mathrm{F}_{\mathrm{abs}}\right)$ is the number of pulses with a fluence $F_{\text {abs }}$.

In our recent investigations [7] we showed that the nature of single-shot XUV-induced ablation of Ru is photomechanical spallation in the stress confinement regime. The latter means that heating of the lattice is faster than the acoustic relaxation time, which means that heating occurs at almost isochoric conditions. It was shown in damage studies of metals induced with optical lasers [18-20] that this situation leads to the generation of large thermo-induced stresses 
and, as a result, to spallation of the top part of a metal. The single-shot spallation threshold at $20^{\circ}$ grazing incidence was measured to be $F_{\text {spall }}=64+/-13 \mathrm{~mJ} / \mathrm{cm}^{2}$ of absorbed fluence, while the melting threshold was calculated to be $F_{\text {melt }}=13 \mathrm{~mJ} / \mathrm{cm}^{2}$ [7]. It is known from literature [18], that spallation starts with nucleation of subsurface voids or cavities in a melted layer of irradiated metal, created as a result of propagation of a tensile stress wave. In a particular fluence range high enough to cause melting, but not sufficient to induce complete spallation, the cavities can remain frozen below the surface, which was proven experimentally [19-21] and with molecular dynamic simulations [19]. Therefore, for metals there is a cavitation threshold that is lower than the spallation threshold.

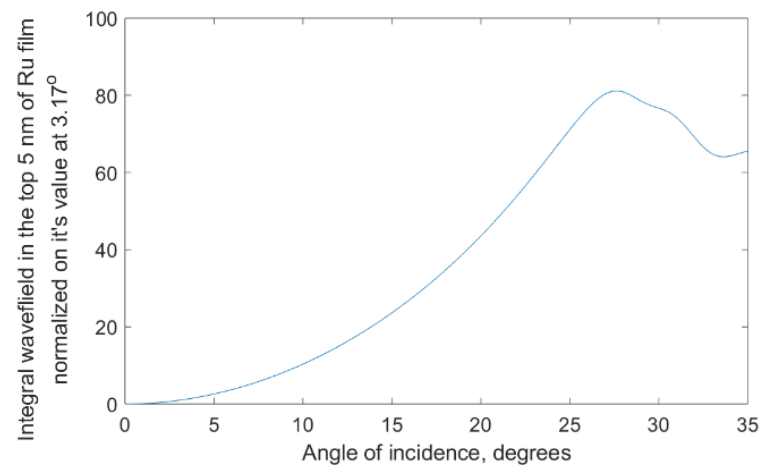

Fig. 4 The integral of the absorbed energy density in the top $5 \mathrm{~nm}$ of the Ru film on the angle of incidence, normalized to its value at $3.17^{\circ}$.

Within this damage mechanism, we suggest that individual pulses with the highest fluence in a pulse train are capable of not only melting the surface of $\mathrm{Ru}$, but causing cavitation below the surface, as described above. The existence of subsurface cavities can create significant roughness and swelling of the surface [19-21].

This hypothesis can also explain the difference in damage caused by multiple pulses at $3.17^{\circ}$ and $20^{\circ}$ grazing incidence (Fig. 2). The angular dependence of the absorbed energy, simulated as the integral of the electromagnetic field (EM) in the top $5 \mathrm{~nm}$ of the film [22], normalized to its value at $3.17^{\circ}$ is shown in Fig. 4. In the region of total external reflection, for $\mathrm{Ru}$ for 13.5 $\mathrm{nm}$ light ranging from $0^{\circ}$ to $27^{\circ}$ grazing incidence, only evanescent waves penetrate into the film [23]. The penetration depth in our case is about $3 \mathrm{~nm}$. However, the density of the EM field increases with increasing angle of incidence and, therefore, the absorption dose also increases. The increased absorbed fluence may eventually reach the spallation threshold, resulting in locally occurring spallation of $\mathrm{Ru}$.

The difference in the damage caused at very grazing and close to critical angles of incidence can be explained assuming the cavitation to be the onset of damage. The atomic force microscopy (AFM) image of the surface damaged by 209 pulses from Fig. 2(b) is shown in Fig. 5.

In a first approximation, the roughness observed in Fig. 5, can be considered as surface areas inclined at a certain angle $\theta *$ to the prime film surface. Therefore, the effective incident angle at the surface is not $\theta$ but $\theta+\theta^{*}$ and therefore the absorption dose is locally increased. According to Fig. 4, the same increment $\theta^{*}$ of the incidence angle will cause much more increase in the absorbed dose for less grazing angles of incidence. For $3.17^{\circ}$ prime incidence angle $\theta$, an increase of the angle of incidence, for example by $\theta^{*}=20^{\circ}$ as shown in Fig. 5, will lead to an increase of the absorbed dose by a factor of 70 , what for fluence of $15 \mathrm{~mJ} / \mathrm{cm}^{2} \mathrm{means}$ locally an increase to $1 \mathrm{~J} / \mathrm{cm}^{2}$, that is much more than spallation threshold. However, for an incidence angle of $\theta=20^{\circ}$ similar inclination will increase the absorbed dose by not more than $50 \%$, leading to an effective maximum fluence of $45 \mathrm{~mJ} / \mathrm{cm}^{2}$ for the pulse with the highest 
fluence in the histogram in Fig. 3. This is still below the spallation threshold for a pristine Ru film.

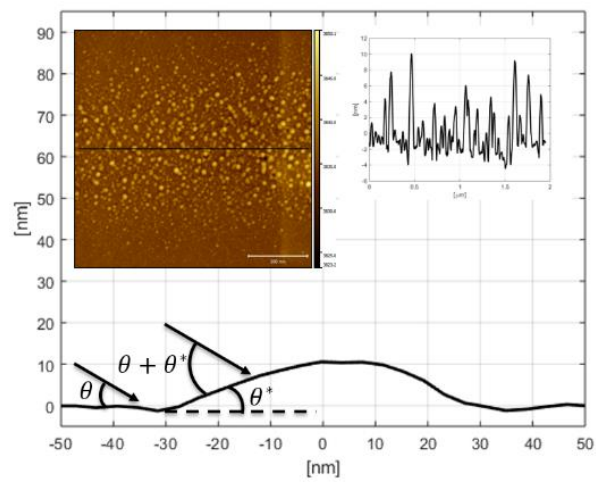

Fig. 5. The AFM image of damage caused by 209 pulses. The black line on the AFM image (top left) indicates the cross-section of entire scan shown in top right corner. The magnified crosssection of the height of one swollen region (bottom plot) shows that due to swelling the incidence angle can be increased by $\theta^{*}=20^{\circ}$.

\section{Conclusions}

We present an experimental study of the damage caused by multiple ultra-short XUV pulses in $\mathrm{Ru}$ coatings. We found that the multi-shot damage threshold does not depend on the repetition rate by comparing damage threshold values obtained for 100 pulses arriving at $3.17^{\circ}$ with repetition rate ranging from $250 \mathrm{KHz}$ to $1 \mathrm{MHz}$.

Based on analysis of the development of damage morphology caused by an increase in the number of pulses arriving at $20^{\circ}$ grazing angle and with a fluence close to the damage threshold, we suggest that the prime cause of the multi-shot damage is roughening of the Ru surface induced by a cavitation process. Therefore, multi-shot damage threshold should be equal to the cavitation threshold.

Comparing the morphology of the craters created pulses arriving at $3.17^{\circ}$ and $20^{\circ}$ grazing incidence we arrive at the conclusion that surface roughening should lead to local increase in the absorption of radiation, which may subsequently lead to local spallation of the roughened areas in $\mathrm{Ru}$ films.

\section{Funding and acknowledgments}

\section{Funding}

Industrial Focus Group XUV Optics of the MESA+ Institute for Nanotechnology of the University of Twente, notably the industrial partners ASML, Carl Zeiss SMT GmbH, Malvern Panalytical, as well as the Province of Overijssel and the Foundation FOM. We acknowledge The Dutch Topconsortia Kennis en Innovatie (TKI) program on high-tech systems and materials (grant No. 14 HTSM 05), the Polish National Science Center (grant No. DEC2011/03/B/ST3/02453; grant No. DEC-2012/06/M/ST3/00475), the EU FP7 EAGLE project co-financed by the Polish Ministry of Science and Higher Education (grant No. REGPOT-CT2013-316014), the Czech Science Foundation (grant No. 14-29772S; grant No. 17-05167S), and the Ministry of Education, Youth and Sports of the Czech Republic (grant No. LTT17015; grant No. CZ.02.1.01/0.0/0.0/16_013/0001552) for financial support.

\section{Acknowladgments}


Support from the operators of the FLASH facility is gratefully acknowledged. Furthermore we acknowledge the support of the Furthermore we acknowledge the help of Jana Buchheim and Klaus Mann.

\section{References}

1. I. Inoue, Y. Inubushi, T. Sato, K. Tono, T. Katayama, T. Kameshima, K. Ogawa, T. Togashi, S. Owada, Y. Amemiya, T. Tanaka, T. Hara, and M. Yabashi, "Observation of femtosecond X-ray interactions with matter using an X-ray-X-ray pump-probe scheme," Proceedings of the National Academy of Sciences 113, 1492-1497 (2016).

2. J. Krzywinski, R. Conley, S. Moeller, G. Gwalt, F. Siewert, C. Waberski, T. Zeschke, and D. Cocco, "Damage thresholds for blaze diffraction gratings and grazing incidence optics at an X-ray free-electron laser," Journal of Synchrotron Radiation 25, 85-90 (2018).

3. J. Krzywinski, D. Cocco, S. Moeller, and D. Ratner, "Damage threshold of platinum coating used for optics for self-seeding of soft x-ray free electron laser," Optics Express 23, 5397-5405 (2015).

4. S. P. Hau-Riege, R. A. London, A. Graf, S. L. Baker, R. Soufli, R. Sobierajski, T. Burian, J. Chalupsky, L. Juha, J. Gaudin, J. Krzywinski, S. Moeller, M. Messerschmidt, J. Bozek, and C. Bostedt, "Interaction of short x-ray pulses with low-Z x-ray optics materials at the LCLS free-electron laser," Optics Express 18, 23933-23938 (2010)

5. R. Sobierajski, I. Jacyna, P. Dłuzewski, M. T. Klepka, D. Klinger, J. B. Pełka, T. Burian, V. Hájková, L. Juha, K. Saksl, V. Vozda, I. Makhotkin, E. Louis, B. Faatz, K. Tiedtke, S. Toleikis, H. Enkisch, M.

Hermann, S. Strobel, R. A. Loch, and J. Chalupsky, "Role of heat accumulation in the multi-shot damage of silicon irradiated with femtosecond XUV pulses at a $1 \mathrm{MHz}$ repetition rate," Optics Express 24, 1546815477 (2016).

6. I. A. Makhotkin, R. Sobierajski, J. Chalupsky, K. Tiedtke, G. de Vries, M. Stormer, F. Scholze, F. Siewert, R. W. E. van de Kruijs, I. Milov, E. Louis, I. Jacyna, M. Jurek, D. Klinger, L. Nittler, Y. Syryanyy, L. Juha, V. Hajkova, V. Vozda, T. Burian, K. Saksl, B. Faatz, B. Keitel, E. Plonjes, S. Schreiber, S. Toleikis, R. Loch, M. Hermann, S. Strobel, H.-K. Nienhuys, G. Gwalt, T. Mey, and H. Enkisch, "Experimental study of EUV mirror radiation damage resistance under long-term free-electron laser exposures below the singleshot damage threshold," Journal of Synchrotron Radiation 25, 77-84 (2018).

$7 . \quad$ I. Milov, "Nature of Single-Shot Damage of Ru thin films irradiated by femtosecond Extreme UV FreeElectron Laser," Submitted to Optics Express (2018).

8. W. Ackermann and G. Asova and V. Ayvazyan and A. Azima and N. Baboi and J. Bahr and V. Balandin and B. Beutner and A. Brandt and A. Bolzmann and R. Brinkmann and O. I. Brovko and M. Castellano and P. Castro and L. Catani and E. Chiadroni and S. Choroba and A. Cianchi and J. T. Costello and D. Cubaynes and J. Dardis and W. Decking and H. Delsim Hashemi and A. Delserieys and G. Di Pirro and M. Dohlus and S. Dusterer and A. Eckhardt and H. T. Edwards and B. Faatz and J. Feldhaus and K. Flottmann and J. Frisch and L. Frohlich and T. Garvey and U. Gensch and C. Gerth and M. Gorler and N. Golubeva and H. J. Grabosch and M. Grecki and O. Grimm and K. Hacker and U. Hahn and J. H. Han and K. Honkavaara and T. Hott and M. Huning and Y. Ivanisenko and E. Jaeschke and W. Jalmuzna and T. Jezynski and R. Kammering and V. Katalev and K. Kavanagh and E. T. Kennedy and S. Khodyachykh and K. Klose and V. Kocharyan and M. Korfer and M. Kollewe and W. Koprek and S. Korepanov and D. Kostin and M. Krassilnikov and G. Kube and M. Kuhlmann and C. L. S. Lewis and L. Lilje and T. Limberg and D. Lipka and F. Lohl and H. Luna and M. Luong and M. Martins and M. Meyer and P. Michelato and V. Miltchev and W. D. Moller and L. Monaco and W. F. O. Muller and O. Napieralski and O. Napoly and P. Nicolosi and D. Nolle and T. Nunez and A. Oppelt and C. Pagani and R. Paparella and N. Pchalek and J. Pedregosa Gutierrez and B. Petersen and B. Petrosyan and G. Petrosyan and L. Petrosyan and J. Pfluger and E. Plonjes and L. Poletto and K. Pozniak and E. Prat and D. Proch and P. Pucyk and P. Radcliffe and H. Redlin and K. Rehlich and M. Richter and M. Roehrs and J. Roensch and R. Romaniuk and M. Ross and J. Rossbach and V. Rybnikov and M. Sachwitz and E. L. Saldin and W. Sandner and H. Schlarb and B. Schmidt and M. Schmitz and P. Schmuser and J. R. Schneider and E. A. Schneidmiller and S. Schnepp and S. Schreiber and M. Seidel and D. Sertore and A. V. Shabunov and C. Simon and S. Simrock and E. Sombrowski and A. A. Sorokin and P. Spanknebel and R. Spesyvtsev and L. Staykov and B. Steffen and F. Stephan and F. Stulle and H. Thom and K. Tiedtke and M. Tischer and S. Toleikis and R. Treusch and D. Trines and I. Tsakov and E. Vogel and T. Weiland and H. Weise and M. Wellhofer and M. Wendt and I. Will and A. Winter and K. Wittenburg and W. Wurth and P. Yeates and M. V. Yurkov and I. Zagorodnov and K. Zapfe, "Operation of a free-electron laser from the extreme ultraviolet to the water window," Nat Photon 1, 336-342 (2007).

9. R. Sobierajski, M. Jurek, J. Chalupský, J. Krzywinski, T. Burian, S. D. Farahani, V. Hájková, M. Harmand, L. Juha, D. Klinger, R. A. Loch, C. Ozkan, J. B. Pełka, K. Sokolowski-Tinten, H. Sinn, S. Toleikis, K. Tiedtke, T. Tschentscher, H. Wabnitz, and J. Gaudin, "Experimental set-up and procedures for the investigation of XUV free electron laser interactions with solids," Journal of Instrumentation 8, P02010 (2013).

10. K. Tiedtke, A. Azima, N. v. Bargen, L. Bittner, S. Bonfigt, S. Düsterer, B. Faatz, U. Frühling, M. Gensch, G. Ch, N. Guerassimova, U. Hahn, T. Hans, M. Hesse, K. Honkavaar, U. Jastrow, P. Juranic, S. Kapitzki, 
B. Keitel, T. Kracht, M. Kuhlmann, W. B. Li, M. Martins, T. Núñez, E. Plönjes, H. Redlin, E. L. Saldin, E. A. Schneidmiller, J. R. Schneider, S. Schreiber, N. Stojanovic, F. Tavella, S. Toleikis, R. Treusch, H. Weigelt, M. Wellhöfer, H. Wabnitz, M. V. Yurkov, and J. Feldhaus, "The soft x-ray free-electron laser FLASH at DESY: beamlines, diagnostics and end-stations," New Journal of Physics 11, 023029 (2009).

11. A. Aquila, R. Sobierajski, C. Ozkan, V. Hájková, T. Burian, J. Chalupský, L. Juha, M. Störmer, S. Bajt, M. T. Klepka, P. Dłużewski, K. Morawiec, H. Ohashi, T. Koyama, K. Tono, Y. Inubushi, M. Yabashi, H. Sinn, T. Tschentscher, A. P. Mancuso, and J. Gaudin, "Fluence thresholds for grazing incidence hard Xray mirrors," Appl. Phys. Lett. 106, 241905 (2015).

12. J. M. Liu, "Simple technique for measurements of pulsed Gaussian-beam spot sizes," Opt. Lett. 7, 196198 (1982).

13. J. Chalupský, L. Juha, J. Kuba, J. Cihelka, V. Hájková, S. Koptyaev, J. Krása, A. Velyhan, M. Bergh, C. Caleman, J. Hajdu, R. M. Bionta, H. Chapman, S. P. Hau-Riege, R. A. London, M. Jurek, J. Krzywinski, R. Nietubyc, J. B. Pelka, R. Sobierajski, J. Meyer-ter-Vehn, A. Tronnier, K. Sokolowski-Tinten, N. Stojanovic, K. Tiedtke, S. Toleikis, T. Tschentscher, H. Wabnitz, and U. Zastrau, "Characteristics of focused soft X-ray free-electron laser beam determined by ablation of organic molecular solids," Optics Express 15, 6036-6043 (2007).

14. J. Chalupský, T. Burian, V. Hájková, L. Juha, T. Polcar, J. Gaudin, M. Nagasono, R. Sobierajski, M. Yabashi, and J. Krzywinski, "Fluence scan: an unexplored property of a laser beam," Optics Express 21, 26363-26375 (2013).

15. J. Chalupský, J. Krzywinski, L. Juha, V. Hájková, J. Cihelka, T. Burian, L. Vyšín, J. Gaudin, A. Gleeson, M. Jurek, A. R. Khorsand, D. Klinger, H. Wabnitz, R. Sobierajski, M. Störmer, K. Tiedtke, and S. Toleikis, "Spot size characterization of focused non-Gaussian X-ray laser beams," Optics Express 18, 27836-27845 (2010).

16. F. Scholze, C. Laubis, C. Buchholz, A. Fischer, S. Ploeger, F. Scholz, H. r. Wagne, and G. Ulm, "Status of EUV reflectometry at PTB," Pros. SPIE 5751, 749-758 (2005).

17. K. Tiedtke, J. Feldhaus, U. Hahn, U. Jastrow, T. Nunez, T. Tschentscher, S. V. Bobashev, A. A. Sorokin, J. B. Hastings, S. Möller, L. Cibik, A. Gottwald, A. Hoehl, U. Kroth, M. Krumrey, H. Schöppe, G. Ulm, and M. Richter, "Gas detectors for x-ray lasers," J. Appl. Phys. 103, 094511 (2008).

18. V. V. Zhakhovskii, N. A. Inogamov, and K. Nishihara, "New mechanism of the formation of the nanorelief on a surface irradiated by a femtosecond laser pulse," JETP Letters 87, 423-427 (2008).

19. C. Wu, M. S. Christensen, J.-M. Savolainen, P. Balling, and L. V. Zhigilei, "Generation of subsurface voids and a nanocrystalline surface layer in femtosecond laser irradiation of a single-crystal Ag target," Phys. Rev. B 91, 035413 (2015).

20. S. I. Ashitkov, N. A. Inogamov, V. V. Zhakhovskii, Y. N. Emirov, M. B. Agranat, I. I. Oleinik, S. I. Anisimov, and V. E. Fortov, "Formation of nanocavities in the surface layer of an aluminum target irradiated by a femtosecond laser pulse," JETP Letters 95, 176-181 (2012).

21. J.-M. Savolainen, M. S. Christensen, and P. Balling, "Material swelling as the first step in the ablation of metals by ultrashort laser pulses," Phys. Rev. B 84, 193410 (2011).

22. M. Born and E. Wolf, Principles of Optics, Seventh ed. (Campridge university press, Cambridge, 2000).

23. M. J. Bedzyk, G. M. Bommarito, and J. S. Schildkraut, "X-ray standing waves at a reflecting mirror surface," Physical Review Letters 62, 1376-1379 (1989). 